\title{
Oxidized PLs and Vascular Inflammation
}

\author{
Maceler Aldrovandi • Valerie B. O’Donnell
}

Published online: 20 March 2013

(C) The Author(s) 2013. This article is published with open access at Springerlink.com

\begin{abstract}
Oxidized PLs (OxPLs) generated in health and disease are now recognized as important mediators of cellular signalling. There is an increasing body of evidence showing that PL peroxidation is not only increased in vascular disorders, but is also a physiological event of relevance to coagulation, innate immunity, and self-tolerance. Nonenzymatically formed OxPLs generated during chronic inflammation is an uncontrolled event, generating hundreds of diverse structures, and prone to more deleterious bioactivities. In contrast, enzymatic formation of OxPLs is tightly regulated, involving receptors and intracellular signaling, acting as part of the normal physiological response to injury in order to restore homeostasis. In the present review, the major nonenzymatic OxPLs structures found during vascular inflammation are summarized, along with a brief description of their known biological activities. Also, we review what is currently known about enzymatic formation of OxPLs by acutely activated immune cells and their signaling actions under homeostatic and pathological conditions.
\end{abstract}

Keywords Oxidized PL · Vascular inflammation ·

Atherosclerosis $\cdot$ Lipoxygenase

\section{Introduction}

Phospholipids (PLs) are the major components of cell membranes and play a crucial role in the biochemistry of all living cells. They provide a permeable barrier as well as

This article is part of the Topical Collection on Vascular Biology

M. Aldrovandi · V. B. O’Donnell $(\bowtie)$

Institute of Infection and Immunity, School of Medicine,

Cardiff University, Heath Park,

Cardiff CF14 4XN, UK

e-mail: o-donnellvb@cardiff.ac.uk

M. Aldrovandi

e-mail: aldrovandim@cardiff.ac.uk acting as substrates for the synthesis of essential signalling mediators such as eicosanoids. PLs consist of a glycerol backbone connected to two nonpolar fatty acids at sn 1 and sn2 and a phosphate-containing polar headgroup at the sn3 position. In mammalian cells, polyunsaturated fatty acids are usually bound to the sn2 of glycerol where the majority of oxidation will occur. Oxidation of esterified polyunsaturated fatty acids can be initiated via both enzymatic and nonenzymatic mechanisms, and can form hundreds of diverse structures $[1 \bullet \bullet, 2]$. Phosphatidylcholine (PC) is the most abundant PL species in eukaryotic cell membranes (40-50 \%) and, as a result, the vast proportion of nonenzymaticallyformed OxPLs detected in mammalian tissues contain the choline headgroup. In contrast, phosphatidylethanolamine (PE) is the prominent PL class modified via enzymatic oxidation [3-7].

OxPLs exhibit a vast range of biological activities, including acting as markers of "modified-self" that can induce apoptotic and senescent cell removal, and more complex interactions, such as regulation of immune responses $[1 \bullet \cdot 2]$. Their involvement in activation of platelet aggregation and monocyte adhesion, as well as suppression of cytokine generation and neutrophil superoxide release has been reported [8-12]. Also, they can act as both pro- and anti-inflammatory mediators during the progression of atherosclerosis.

Nonenzymatically oxidized PLs that derive from PL hydroperoxide decomposition have been measured in atherosclerotic lesions of humans and other mammalian models of atherosclerosis $[1 \bullet \bullet, 13-17]$. LC-MS analysis of human atheroma at different stages of development identified similar OxPL species at different stages of the disease [18]. The detection of early oxidation species, like hydroperoxides and further oxidized products such as fragmented OxPLs, suggests that during the development of atherosclerotic lesions, nonenzymatically formed OxPLs are continuously being formed through hydroperoxide decomposition. Even though this is expected to be an uncontrolled event, whether the initial hydroperoxides originate from oxidation reactions 
driven by enzymatic or nonenzymatic processes in vivo remain unknown.

Enzymatically formed OxPLs are generated by proteins that are conserved among all mammalian species, such as lipoxygenases (LOX) and cyclooxygenases (COX), and their synthesis involves receptors and intracellular signalling pathways $[1 \bullet \bullet, 19 \bullet \bullet$. Tight control of this process and associated biological actions of the products observed in vitro, indicates that these lipids are of physiological importance and likely to play a role in innate immune defences and haemostasis (see below). In contrast, nonenzymatic oxidized PLs are generated in diseases characterized by chronic inflammation, such as atherosclerosis, where the enhanced production of free radicals, as a result of immune cells activation, leads to uncontrolled oxidant toxicity. In both circumstances, common bioactivities are displayed with both classes likely being important in human health and disease.

In this review, we summarise the major nonenzymatic OxPL structures found during vascular inflammation and we give a brief description of their known biological activities. Then, we focus on what is known about enzymatic formation of OxPLs generated by acutely activated immune cells and their signaling actions in health and disease.

\section{Nonenzymatic Formation of Oxidized PLs}

Atherosclerosis is a chronic vascular disorder characterized by an accumulation of lipids in the artery wall and persistent inflammation [20•]. The initiation and progression of the disease is controlled by inflammatory molecular and cellular mediators, which often trigger oxidative damage to biological molecules. Enhanced production of free radicals including reactive oxygen species (ROS) generated by activated phagocytes results in increased levels of OxPLs, which have been detected in human atherosclerotic lesions [13, 21].

Nonenzymatic formation of oxidized PLs is regulated by chemical reaction mechanisms that are propagated by either nonradical reactive oxygen species (ROS) or free radicals. Depending on the reactive oxygen species and the nature of the lipid target, both nonradical ROS and free radicals can generate a diverse range of lipid structures without enzymatic regulation $[22,23]$. However, how this form of oxidation is initiated is currently unknown and could include either enzymatic or nonenzymatic mechanisms.

Relevant nonenzymatic oxidative modifications to PLs in terms of inflammatory cell biology rely greatly on the nature of the oxidant species, such as those mediated by ROS like nitrogen dioxide $\left(\mathrm{NO}^{2}\right)$, nitric oxide, superoxide, hydrogen peroxide, hydroxyl radical $\left({ }^{\circ} \mathrm{OH}\right)$ hypochlorite $(\mathrm{HOCl})$, and singlet $\mathrm{O}^{2}[24,25]$. These are often generated by activated phagocytes during chronic inflammation. In addition, reactive oxygen species such as oxides of nitrogen and reactive aldehydes can similarly originate from multiple exogenous sources, including air pollution, environment, smoking, UV light, or ionizing radiation.

Inorganic radical species such as ${ }^{\circ} \mathrm{OH}$ and ${ }^{\circ} \mathrm{NO}_{2}$ can react with various unsaturated lipids, leading to the formation of isoprostanes, oxidized cholesterol, and other lipid species $[26,27]$. PLs containing polyunsaturated fatty acids are very susceptible to peroxidation by reactive oxygen species due to the presence of bisallylic methylene groups between double bonds. Hence, hydrogens become more susceptible to abstraction in PLs containing these lipids. The initial reaction of inorganic radicals with polyunsaturated fatty acids involves hydrogen abstractions of the bisallylic hydrogens, followed by rearrangement of double bonds and addition of oxygen, which leads to highly reactive peroxyl radicals and subsequently formation of hydroperoxides $[1 \bullet \cdot]$. Also, Fenton-like reactions, where lipid peroxyl or alkoxyl radicals are produced by decomposition of preformed lipid hydroperoxides can act as the initial mechanism of PL oxidation [28].

Following the initiation step, the resulting peroxyl radicals or hydroperoxides then can propagate forming secondary peroxidation products through a variety of mechanisms, without participation of enzymes. The three major pathways involve (1) cyclization, rearrangement, and further oxidation reactions via the isoprostane pathways (e.g., isoprostanes, isolevuglandins, and isothromboxanes); (2) hydroperoxide fragmentation generating shortened fatty acid residues [e.g., oxidatively truncated (un)saturated PLs]; (3) additional oxidation (e.g., dihydroxy-PC and epoxy-monohydroxy-PC) $[1 \bullet \cdot]$.

During vascular inflammation LDL molecules accumulated within the subendothelial space undergo oxidative modification, resulting in the formation of mildly modified LDL (MM-LDL) and oxidized LDL (ox-LDL). 1-Palmitoyl2-arachidonoyl-sn-glycero-3-phosphorylcholine (PAPC), a naturally occurring PL found in cell membranes, is one the main PLs present in MM-LDL. Oxidation of PAPC induced by free radicals results in formation of a range of products, including fragmented (e.g., truncated PLs) and nonfragmented OxPLs (e.g., isoprostanes, levuglandins, isofurans, and isothromboxanes), which are described in the following sections.

\section{Fragmented OxPLs}

Fragmented OxPLs are generated via oxidative fragmentation of hydroperoxides, followed by transformation of peroxides/peroxyls into advanced oxidation products $[1 \bullet \bullet]$. Oxidative fragmentation of peroxyl radicals forms truncated PLs such as PAPC-derived truncated oxidized products, 1palmytoyl-2-(5-oxovaleroyl)-sn-glycero-3-phosphocholine (POVPC), 1-palmitoyl-2-glutaroyl-sn-glycero-3- 
phosphocholine (PGPC), 5-keto-6-octendioic acid ester of 2-lyso-phosphocholine (KOdiA-PC), 5-hydroxy-8-oxo-6octenedioic acid ester of 2-lysophosphocholine (HOdiAPC), 1-palmitoyl-2-(5-hydroxy-8-oxooct-6-enoyl)-snglycero-3-phosphocholine (HOOA-PC) and 5-keto-8-oxo6-octenoic acid esters of 2- lysophosphocholine (KOOAPC), which have been found at high concentrations within fatty streaks of cholesterol-fed rabbits and hyperlipidemic mice [16, 17]. Quantification of POVPC and PGPC in aortae of cholesterol-fed rabbits demonstrated approximately $60 \mu \mathrm{g} / \mathrm{g}$ and $45 \mu \mathrm{g} / \mathrm{g}$ of wet tissue weight, respectively [17]. In lesions of cholesterol-fed rabbits these levels were even higher, approximately $100 \mu \mathrm{g} / \mathrm{g}$ of POVPC and $60 \mu \mathrm{g} / \mathrm{g}$ of wet tissue weight in PGPC [13].

Oxidative cleavage of PAPC forms POVPC, which can be further oxidized to PGPC. Although POVPC and PGPC are of similar chemical structure they mediate distinct activities - for instance, POVPC enhances neutrophil binding to endothelial cells, while PGPC inhibits [11]. A recent study using gene expression analysis has shown that OxPAPC, accumulated in atherosclerotic lesions, is proatherogenic and regulates the expression of more than a thousand genes in human aortic endothelial cells [29]. POVPC and PGPC have multiple effects on different cell types, including regulation of inflammatory functions of endothelial cells, inhibition of macrophage ox-LDL uptake, induction of apoptotic pathways in vascular smooth muscle cells, increased monocyte synthesis of chemotactic factors, and enhanced surface expression of adhesion molecules on endothelial cells [1・•, 11, 13, 30-34]. In addition, KOdiA-PC, HOdiA-PC, HOOA-PC, and KOOA-PC formed from fragmentation of hydroperoxide-derived dienes can act as ligands for the scavenger receptor CD36 [16]. HOOA-PC induces proinflammatory activities by promoting monocyte access to chronic lesions, as seen in atherosclerosis, via endothelial cell/monocyte binding and increasing chemokine (MCP-I and IL-8) levels [35].

\section{Non-Fragmented OxPLs}

Isoprostanes are prostaglandin-like compounds formed in situ in cell membranes via free radical-mediated peroxidation of polyunsaturated fatty acids, mainly arachidonic acid $[1 \bullet \bullet]$. The side chains of these molecules are almost exclusively oriented cis to the cyclopentane ring with several possible regioisomers yielding a large variety of structures. Unlike prostaglandins, isoprostane synthesis is believed to occur independently of cyclooxygenases. Their formation is initiated via peroxidation of arachidonic acid induced by free radicals, generating arachidonyl radicals containing two molecules of oxygen [36, 37].

Nonregioselective abstraction of hydrogen atoms from arachidonic acid forms three regioisomeric pentadienyl radicals which react with oxygen molecules, generating four $\mathrm{PGH}_{2}$-like intermediates. The bicyclic endoperoxide intermediates are subsequently reduced to form $\mathrm{PGF}_{2}$-like compounds or go through various rearrangements to form isothromboxanes and other oxidized lipids [2].

Differently to prostaglandins that result from enzymatic oxidation of free arachidonic acid, isoprostanes are thought to be primarily generated via nonenzymatic oxidation of PLesterified arachidonic acid [38]. Esterified isoprostanes are then released as free acids by either phospholipase $\mathrm{A}_{2}$ or platelet-activating factor acetylhydrolase activity, into the circulation. Eight-epi $\mathrm{PGF}_{2 \alpha}$ and $\mathrm{IPF}_{2 \alpha}$-I are examples of isoprostanes found at high concentration in human atherosclerotic lesions, localized intracellularly, predominantly in macrophage-rich areas [39].

Separate to the transformation of bicyclic endoperoxides, is formation of 1-palmitoyl-2-(5,6-epoxyisoprostane E2)-snglycero-3-phosphocholine (PEIPC). This oxidised PL is formed through free radical oxidation and cyclization of the arachidonic acid group of PAPC, followed by dehydration of the isoPGG $\mathrm{PGC}_{2}-\mathrm{PC}$ to produce epoxyisoprostane-PLs [40]. Further dehydration of the cyclic isoprostane ring of PEIPC generates 1-hexadecanoyl-2-(5,6-epoxyisoprostane A2 oyl)-snglycero-3 phosphocholine (PECPC). PEIPC was detected at high levels in atherosclerotic lesions of cholesterol-fed rabbits, $60-100 \mu \mathrm{g} / \mathrm{g}$ of wet tissue weight $[13,16]$. PEIPC and PECPC are potent activators of endothelial cells, increasing synthesis of IL-8 and MCP-1, and are also concentrated within atherosclerotic lesions [10, 11, 31, 41].

Another example of nonfragmented OxPLs are esterified isolevuglandins formed via rearrangement of iso $\mathrm{PGH}_{2}-\mathrm{PC}$, which differ from isoprostanes by containing aldehyde groups, making them extremely reactive toward amine-containing molecules, such as proteins. Hence, isolevuglandins have been found as protein adducts in vivo. They are detected in plasma at significantly higher levels compared to isoprostanes and, considering that many proteins have half-lives varying from days to weeks, isolevuglandin-protein adducts can accumulate over time and possibly be used to assess oxidative stress status in vivo [42]. For example, Poliakov and coworkers reported highly elevated isolevuglandins levels in plasma in individuals with atherosclerosis compared to controls [42].

Similarly to isoprostanes, isofurans are nonenzymatically formed but contain a substituted tetrahydrofuran ring. High oxygen concentrations favour isofuran over isoprostane formation. Hence, production of isofurans is differentially modulated by oxygen levels [43]. Due to their stability, esterified isofurans can be used as an index of lipid oxidation.

Last, $\mathrm{PGH}_{2}$-like bicyclic endoperoxide intermediates can be transformed through spontaneous rearrangement into 
esterified isothromboxane $A_{2}$, which is very unstable and is rapidly hydrolysed into isothromboxane $\mathrm{B}_{2}$.

\section{Enzymatic Formation of Oxidized PLs}

Enzymatic formation of oxidized PLs is mediated either by direct PL oxidation or esterification of preformed oxidized fatty acids into PLs. During immune cell activation, OxPLs can be formed via lipoxygenase (LOX) or cyclooxygenase (COX) on both esterified and free polyunsaturated fatty acids $[44,45]$. For instance, phospholipase $\mathrm{A}_{2}\left(\mathrm{PLA}_{2}\right)$ hydrolyses PLs at sn2, generating arachidonate for LOX and COX enzymatic oxidation, forming hydroxyeicosatetraenoic acids (HETEs) and $\mathrm{PGH}_{2}$, respectively [44]. PGH2 then undergoes further enzymatic metabolism to form more stable compounds such as prostaglandin $\mathrm{E}_{2}\left(\mathrm{PGE}_{2}\right), \mathrm{PGD}_{2}$ and thromboxane $\mathrm{B}_{2}$ $\left(\mathrm{TXB}_{2}\right)$. Over the last 6-7 years, it has been observed that oxidized fatty acids like 12-HETE and 15-HETE, and also $\mathrm{PGE}_{2}$, are not only acutely generated as free acids but also as larger PL esterified species with distinct biological and physical properties. These lipids are described in detail in the following sections.

Oxidized PLs Generated by Human Monocytes and Murine Macrophages

Human peripheral monocytes express high levels of the 15LOX isoform that generates 15-HETE, in response to Th2 cytokines. Similarly, naïve murine peritoneal macrophages express the functional equivalent 12/15-LOX, which generates primarily 12 -HETE. The enzyme is constitutively active under basal conditions in both human monocytes and murine macrophages, but can be further activated using ionophore [46].

The Th2 cytokines IL-4 and IL-13 are known to contribute to severity of asthma and lung allergy by inducing ciliated epithelial cell differentiation into mucus producing goblet cells. Studies have shown that IL-4 and IL-13 induce high levels of 15-LOX expression in human asthmatic epithelial cells [47-50]. Zhao and coworkers have reported that human bronchial epithelial cells, in culture with IL-13stimulated 15-LOX expression, generate two 15-HETEPEs, which can enhance expression of MUC5AC, a gene associated with hypersecretion of mucus in asthma [51]. Moreover, in human bronchial epithelial cells, IL-13 induced 15-LOX and its product 15-HETE-PE to bind to PE-binding protein-1, causing its dissociation from Raf-1 and allowing the activation of ERK [52].

More recently, Uderhardt and colleagues showed that OxPLs on the surface of resident peritoneal macrophages serve as binding sites for soluble receptors for apoptotic cells. In this study, 12/15-LOX-expressing resident peritoneal macrophages were reported as responsible for noninflammatory clearance of apoptotic cells and inhibiting their uptake by freshly recruited inflammatory monocytes during inflammation [53••]. Oxidized PE was found to mediate these effects through binding MFG-E8, suggesting that 12/15-LOX enzyme is the major factor orchestrating the regulation of apoptotic cell clearance and preservation of immunologic tolerance [53••].

We previously identified a family of four esterified 15-HETEs, comprising one diacyl (18:0a/15-HETE-PE) and three plasmalogen PE species (18:0p, 18:1p, 16:0p/15-HETE-PE) generated equally in human peripheral monocytes and by murine resident macrophages, both basally, and on activation with calcium ionophore [54]. Without activation, human monocytes already generate basal levels of HETE-PEs, $0.5-3 \mathrm{ng} / 10^{6}$ monocytes. These levels approximately double on ionophore activation $\left(\sim 7 \mathrm{ng} / 10^{6}\right.$ monocytes $)$, suggesting that the LOX enzyme is already constitutively active, generating HETE-PEs without inflammatory stimulation and that the PE-esterified HETEs are executing homeostatic functions [3, 54]. In contrast to free 15-HETE, these products remain cell associated, suggesting an autocrine mode of action. The predominance of the 15S-HETE enantiomer was demonstrated by MS/MS and chiral chromatography, confirming their enzymatic generation. In addition, consistent with the already known ability of the 15-LOX isoform to directly oxidize PLs, stable isotope labelling studies revealed that HETE-PEs are formed by direct oxidation of the intact PE by 15 LOX enzyme, with no requirement for hydrolysis of arachidonate by phospholipases [3, 54]. They correspond to approximately $30 \%$ of the total 15-HETE generated [3].

More recently, two additional families of related lipids have been identified in human IL-4 monocytes and murine peritoneal macrophages - specifically, hydroperoxyeicosatetraenoic acid (HpETE) and ketoeicosatetraenoic acids (KETE) attached to PE [4••]. As for HETE-PEs, they contain positional isomers matching cellular LOX isoform expression, with 15- and 12isomers detected in human or murine cells, respectively. Using LC/MS/MS, the PEs attached to KETEs were identified as 18:0a, 18:0p, 18:1p and 16:0p, where one is acyl-linked and three are plasmalogen-linked at sn1. Although their amounts are lower compared to HETE-PEs $\left(478 \mathrm{pg} / 2 \times 10^{6}\right.$ monocytes) they are also formed on ionophore activation, and are absent in macrophages from 12/15-LOX deficient mice. Consistent with previous reports showing that 12/15$\mathrm{LOX}^{-} /$macrophages are incapable of mounting a normal phagocytic response in vitro [55], we recently observed that they contain larger numbers of cytoplasmic vesicles and anomalous mitochondria, which may allow speculation about defective autophagy or exosomal processing (V. Hammond and V.B.O., unpublished data, 2012). 
We propose that HpETE-PEs are first formed by LOX enzyme, subsequently reduced to HETE-PEs by cellular glutathione peroxidises, and finally HETE-PEs are oxidized by prostaglandin dehydrogenase (PGDH) to KETE-PEs, which remain cell-associated. Experiments using $\mathrm{H}_{2}{ }^{18} \mathrm{O}$ and a 15-PGDH inhibitor suggest that 15-KETE-PEs may form through direct oxidation of preformed HETE-PE by the enzyme, rather than esterification of newly generated KETE.

In terms of biological function, both 15-HETE-PE and 15-KETE-PE dose dependently activate PPAR $\gamma$ transcriptional activity in macrophages. It is speculated that these lipids may act indirectly, either through $\mathrm{PLA}_{2}$ hydrolysis to release free PPAR $\gamma$ ligands, or via upstream modulation of PPAR $\gamma$ expression [4••]. 15HETE-PE can attenuate inflammatory signalling through TLR4 inhibition, a known inhibitor of PPAR $\gamma$ expression and activity $[3,56]$. In this way, dampening down TLR4 via CD14 and LPS-binding protein may result in elevated PPAR $\gamma$ signalling. These findings are consistent with previous reports showing that oxidized free fatty acid generated by LOXs can activate PPAR $\gamma$ [57]. Figure 1 shows the chemical structures of 15-HETE-PEs and 15-KETE-PEs generated by activated human monocytes and their known biological actions.

Thus, new families of bioactive mediators from the 12/15-LOX pathway, as described above, may contribute to its known anti-inflammatory actions in vivo and maybe of potential therapeutic benefit in treating chronic inflammation.

Oxidized PLs Generated by Human Platelets

The contribution of platelets to atherosclerosis has been firmly established over the years. Human platelets express two different enzymes that are known to play a role in PL oxidation, a platelet-specific 12-LOX isoform and COX-1. 12-HETE is the most abundant eicosanoid generated by agonist-activated platelets, but it does not appear to directly regulate platelet function [58]. In contrast, platelet COX-1-derived thromboxane is well known to induce platelet aggregation via the thromboxane receptor and also act as a potent vasoconstrictor [59]. Both enzymes are activated acutely during agonist platelet activation (thrombin, collagen) in a calcium-dependent manner.

We recently showed that agonist-activated human platelets generate six molecular species of esterified 12-HETE, comprising four PEs (16:0p, 18:1p, 18:0p and 18:0a/12HETE-PE) and two PCs (16:0a, 18:0a/12-HETE-PC) via 12-LOX [5••]. Moreover, esterified 12-HETEs are not generated by platelets from $12-\mathrm{LOX}^{-/}$mice (M. Aldrovandi and V.B.O., unpublished data, 2012). The lipids are formed within $5 \mathrm{~min}$ of activation by thrombin, collagen or ionophore, with levels of 12-HETE-PEs reaching $6 \mathrm{ng} / 4 \times 10^{7}$ platelets and 12 -HETE-PC $\sim 18 \mathrm{ng} / 4 \times 10^{7}$ platelets. 12 HETE-PEs and 12-HETE-PC generation is triggered by
Fig. 1 Chemical structures of 15-HETE-PEs and 15-KETE-PEs generated by activated human monocytes

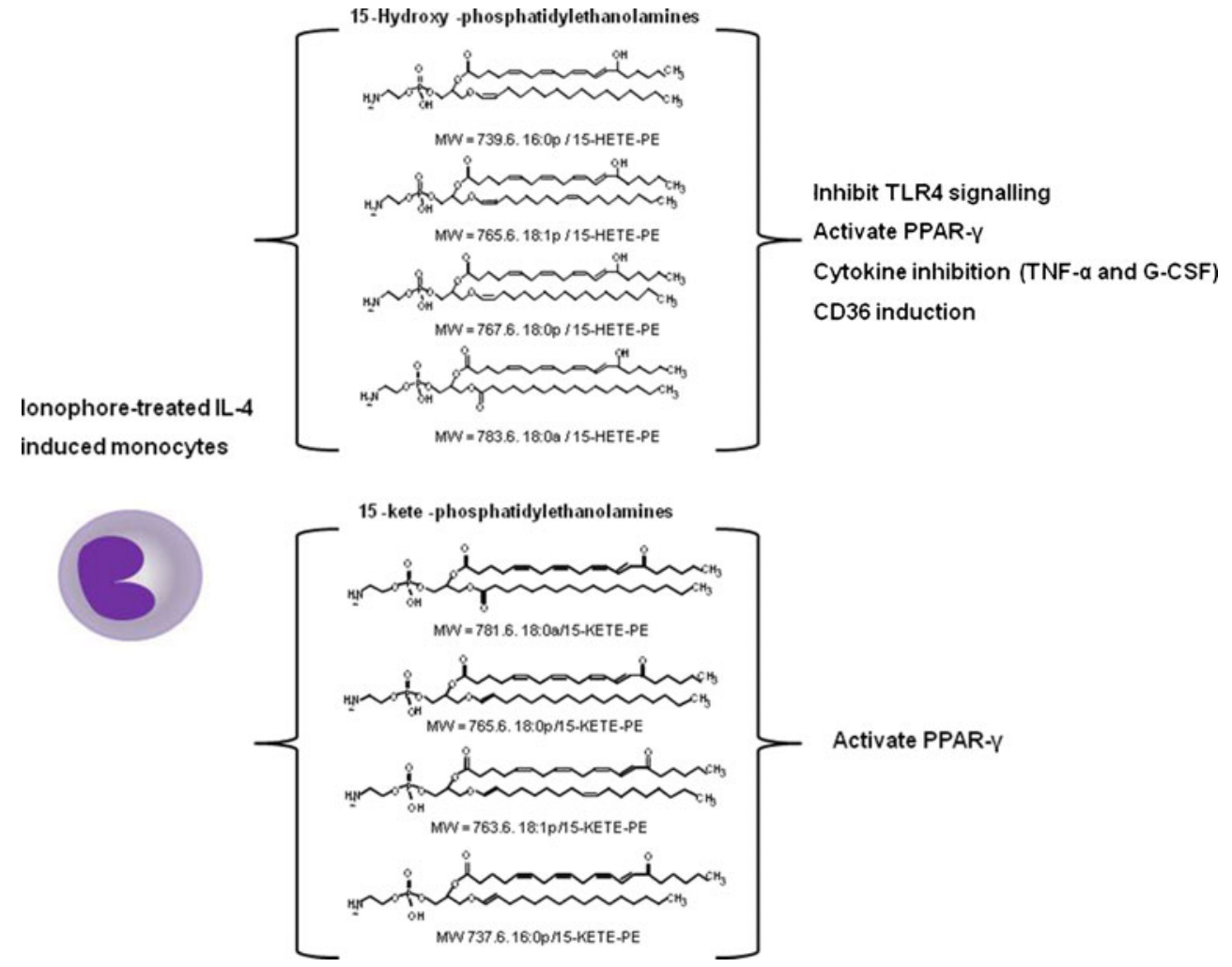


activation of protease activated receptors 1 and 4 (PARs). Several intracellular signalling mediators are required, including $\mathrm{Ca}^{2+}, s r c$ tyrosine kinases, protein kinase $\mathrm{C}$, and $\mathrm{sPLA}_{2}$. Esterified 12-HETEs account for approximately one-third of the total 12-HETE produced. Stable isotope labelling studies showed that 12-HETE-PEs are formed by esterification of preformed 12-HETE, instead of direct PL oxidation [5••]. Esterified 12-HETEs remain predominantly membrane associated, with a proportion of the HETE-PEs trafficking to the outside of the plasma membrane. In accordance with previous observations showing that nonenzymatically oxidised PLs can be procoagulant, esterified 12-HETEs can promote thrombin generation in vitro $[60,61]$.

Activated human platelets also generate several additional PL-esterified hydroxy-fatty acids via 12-lipoxygenase, where the 12-HETE is replaced by 12-LOX-oxidized docosahexaenoic acid (DHA)-containing PE. The products comprise 4 PE-esterified hydroxydocosa-hexaenoic acids (HDOHE-PE), two diacyl (16:0a, 18:0a) and two plasmalogen (16:0p, 18:0p), containing predominantly the 14-HDOHE positional isomer [6•]. They form acutely in the presence of thrombin in a calcium-dependent manner, with levels up to $2-4 \mathrm{ng} / 4 \times 10^{7}$ platelets. Studies using recombinant human 12-LOX and $\mathrm{CPLA}_{2}$ inhibitors demonstrate that formation of HDOHE-PEs occurs via $\mathrm{PLA}_{2}$ hydrolysis of DHA, followed by 12-LOX oxidation and re-esterification of free 14-HDOHE into PLs [6•].

Interestingly, we recently identified COX-1-derived PE esterified $\mathrm{PGE}_{2}$ and $\mathrm{PGD}_{2}$, which are formed within 25 min of platelet activation by thrombin, collagen or ionophore in a calcium dependent manner (M. Aldrovandi and V.B.O., unpublished data, 2012). Their functions remain unknown and generation of PL-esterified prostaglandins in COX-2 expressing cells have not yet been studied. Nevertheless, since synthesis of glyceryl- $\mathrm{PGE}_{2} / \mathrm{D}_{2}$ in response to lipopolysaccharide by macrophages expressing the inducible COX-2 isoform have already been shown, it is also likely that PL-esterified prostaglandins are generated in COX-2-expressing cells [62-64].

\section{Oxidized PLs Generated by Human Neutrophils}

Neutrophils are the most abundant white cells in the circulation and until recently, received little attention for their role in the pathophysiology of atherosclerosis. Nevertheless, studies have provided convincing evidence for the presence of these cells in atherosclerotic plaques [65, 66]. Neutrophils express the 5-LOX isoform, which, upon activation, generates 5-HpETE through oxygenation of arachidonic acid. 5HpETE can undergo further processing to 5-HETE, 5-oxoETE and the potent chemoattractant leukotriene $\mathrm{B}_{4}$. Recently, we reported that primary human neutrophils acutely generate four 5-LOX-derived lipids, comprising plasmalogen and diacyl PL esterified 5-HETEs, in response to bacterial peptides, chemokines, and chemical stimuli, such as phorbol and calcium ionophore [7••]. Four are generated: 16:0a/5-HETE-PC, 18:0p/5-HETE-PE, 18:1p/5HETE-PE, and 16:0p/5-HETE-PE. Upon agonist activation, they form within 2 min with total 5-HETE-PLs reaching up to $0.37 \mathrm{ng} / 10^{6}$ neutrophils. As with platelets, esterified 5HETEs are generated by fast esterification of newly formed free 5-HETE, remaining cell associated and localized to nuclear and extranuclear membrane. They are synthesised via a highly coordinated mechanism, involving receptors and intracellular signalling intermediates, including $\mathrm{Ca}^{2+}, \mathrm{PLC}, \mathrm{cPLA}_{2}, \mathrm{PLA}_{2}$, mitogen-activated protein kinase/extracellular signal-regulated kinase 1 , and 5-LOX activating protein (FLAP). The 5-HETE-PEs regulate a number of neutrophil activities in vitro, including enhancing superoxide and inhibiting neutrophil extracellular trap (NET) release $[7 \bullet \bullet]$.

\section{Conclusion}

OxPLs formed either enzymatically or nonenzymatically are known to have multiple effects on different cell types of relevance to health and inflammatory vascular disease. Several nonenzymatically OxPLs have been identified in atherosclerotic lesions, affecting macrophages, endothelial cells and other cell types, contributing to the initiation and development of the disease.

Over the past few years, it has become clear that OxPLs are important not only during chronic inflammation but also in maintaining homeostasis. In particular, a number of new families of OxPLs enzymatically generated by acutely activated cells have been identified. Their formation is tightly regulated and they remain cell associated, suggesting a paracrine mechanism of action, possibly mediated at or close to the plasma membrane. The great majority of esterified PLs identified in our laboratory are synthesized via LOXs. Nevertheless, in contrast to the idea that prostaglandins are found exclusively as unesterified lipids, we have also observed acute formation of esterified $\mathrm{PGE}_{2} / \mathrm{D}_{2}$-PEs via the constitutively expressed COX-1 isoform, in thrombin activated platelets.

It is clear that formation of nonenzymatic OxPLs during chronic inflammation is an uncontrolled event, originating a diverse range of different OxPLs species. On the other hand, enzymatic formation of OxPLs is tightly regulated, involving receptors and signaling intermediates.

Although significant progress has been made in understanding the formation and function of a number of different esterified PLs, more work is still needed; in particular, a better understanding of the formation, regulation and, and 
interactions of OxPLs in disease would provide insights into the function of lipid mediators, potentially leading to new strategies for prevention and treatment of atherosclerosis, thrombosis, cancer, and other related disorders. In the future, OxPLs may also be used as valuable biomarkers of inflammation, helping in the therapeutic diagnosis.

Conflicts of Interest Maceler Aldrovandi declares no conflicts of interest.

Valerie B. O’Donnell declares no conflicts of interest.

Open Access This article is distributed under the terms of the Creative Commons Attribution License which permits any use, distribution, and reproduction in any medium, provided the original author(s) and the source are credited.

\section{References}

Papers of particular interest, published recently, have been highlighted as:

- Of importance

-. Of major importance

1. .- Bochkov VN, Oskolkova OV, Birukov KG, et al. Antioxid Redox Signal. 2010;12:1009-59. This review comprehensively summarizes the myriad of known oxidized phospholipid species and their biological actions.

2. Fruhwirth GO, Loidl A, Hermetter A. Oxidized phospholipids: from molecular properties to disease. Biochim Biophys Acta. 2007;1772(7):718-36.

3. Morgan AH, Dioszeghy V, Maskrey BH, et al. Phosphatidylethanolamine-esterified eicosanoids in the mouse: tissue localization and inflammation dependent formation in Th-2 disease. J Biol Chem. 2009;284(32):21185-91.

4. •- Hammond VJ, Morgan AH, Lauder SN, et al. Novel ketophospholipids are generated by monocytes and macrophages, detected in Cystic Fibrosis, and activate peroxisome proliferatoractivated receptor- $\gamma$. J Biol Chem. 2012;287(50):41651-66. This study identified the formation of 12-KETE-PEs and 15-KETE-PEs in murine peritoneal macrophages and human monocytes, respectively, which activate PPAR $\gamma$ and may be contribute to the immune regulatory actions of mammalian LOXs in health and disease.

5. •. Thomas CP, Morgan LT, Maskrey BH, et al. Phospholipidesterified eicosanoids are generated in agonist-activated human platelets and enhance tissue factor-dependent thrombin generation. J Biol Chem. 2010;285(10):6891-903. This study identifies six new lipids generated by human platelets that can promote tissue factor dependent coagulation in vitro.

6. • Morgan LT, Thomas CP, Kühn H, O'Donnell VB. Thrombin-activated human platelets acutely generate oxidized docosahexaenoicacid-containing phospholipids via 12-lipoxygenase. Biochem J. 2010;431(1):141-8. This study shows formation of new lipids comprising oxidized docosahexaenoic acid attached to phospholipids by thrombin activated human platelets.

7. • Clark SR, Guy CJ, Scurr MJ, et al. Esterified eicosanoids are acutely generated by 5-lipoxygenase in primary human neutrophils and in human and murine infection. Blood. 2011;117(6):2033-43. This study shows generation of novel esterified eicosanoids generated by human neutrophils in vivo and in vitro.
8. Subbanagounder G, Leitinger N, Shih PT, et al. Evidence that phospholipid oxidation products and/or platelet-activating factor play an important role in early atherogenesis: in vitro and In vivo inhibition by WEB 2086. Circ Res. 1999;85(4):311-8.

9. Androulakis N, Durand H, Ninio E, Tsoukatos DC. Molecular and mechanistic characterization of platelet-activating factor-like bioactivity produced upon LDL oxidation. J Lipid Res. 2005;46(9):192332.

10. Subbanagounder G, Wong JW, Lee H, et al. Epoxyisoprostane and epoxycyclopentenone phospholipids regulate monocyte chemotactic protein-1 and interleukin- 8 synthesis. Formation of these oxidized phospholipids in response to interleukin-1beta. J Biol Chem. 2002;277(9):7271-81.

11. Leitinger N, Tyner TR, Oslund L, et al. Structurally similar oxidized phospholipids differentially regulate endothelial binding of monocytes and neutrophils. Proc Natl Acad Sci U S A. 1999;96(21):12010-5.

12. Blüml S, Rosc B, Lorincz A, et al. The oxidation state of phospholipids controls the oxidative burst in neutrophil granulocytes. J Immunol. 2008;181(6):4347-53.

13. Subbanagounder G, Leitinger N, Schwenke DC, et al. Determinants of bioactivity of oxidized phospholipids. Specific oxidized fatty acyl groups at the sn-2 position. Arterioscler Thromb Vasc Biol. 2000;20:2248-54.

14. Waddington E, Sienuarine K, Puddey I, Croft K. Identification and quantitation of unique fatty acid oxidation products in human atherosclerotic plaque using high-performance liquid chromatography. Anal Biochem. 2001;292(2):234-44.

15. Hoff HF, O'Neil J, Wu Z, et al. Phospholipid hydroxyalkenals: biological and chemical properties of specific oxidized lipids present in atherosclerotic lesions. Arterioscler Thromb Vasc Biol. 2003;23(2):275-82.

16. Podrez EA, Poliakov E, Shen Z, et al. A novel family of atherogenic oxidized phospholipids promotes macrophage foam cell formation via the scavenger receptor CD36 and is enriched in atherosclerotic lesions. J Biol Chem. 2002;277(41):38517-23.

17. Watson $A D$, Leitinger N, Navab M, et al. Structural identification by mass spectrometry of oxidized phospholipids in minimally oxidized low density lipoprotein that induce monocyte/endothelial interactions and evidence for their presence in vivo. J Biol Chem. 1997;272(21): 13597-607.

18. Ravandi A, Babaei S, Leung R, et al. Phospholipids and oxophospholipids in atherosclerotic plaques at different stages of plaque development. Lipids. 2004;39(2):97-109.

19. • O'Donnell VB, Murphy RC. New families of bioactive oxidized phospholipids generated by immune cells: identification and signaling actions. Blood. 2012;120(10):1985-92. This review summarizes the enzymatically formed oxidized phospholipids generated by acutely activated immune cells and it reviews the mass spectrometry methods used for identification of these lipids.

20. Hansson GK, Hermansson A. The immune system in atherosclerosis. Nat Immunol. 2011;12(3):204-12. This review summarizes the involvement of the immune system in atherosclerosis.

21. Berliner JA, Subbanagounder G, Leitinger N, et al. Evidence for a role of phospholipid oxidation products in atherogenesis. Trends Cardiovasc Med. 2001;11(3-4):142-7.

22. Reis A, Spickett CM. Chemistry of phospholipid oxidation. Biochim Biophys Acta. 2012;1818(10):2374-87.

23. Smith WL, Murphy RC. Oxidized lipids formed non-enzymatically by reactive oxygen species. J Biol Chem. 2008;283(23):15513-4.

24. Winterbourn CC, van den Berg JJ, Roitman E, Kuypers FA. Chlorohydrin formation from unsaturated fatty acids reacted with hypochlorous acid. Arch Biochem Biophys. 1992;296(2):547-55.

25. Jerlich A, Pitt AR, Schaur RJ, Spickett CM. Pathways of phospholipid oxidation by $\mathrm{HOCl}$ in human LDL detected by LC-MS. Free Radic Biol Med. 2000;28(5):673-82. 
26. Morrow JD, Minton TA, Mukundan CR, et al. Free radical-induced generation of isoprostanes in vivo. Evidence for the formation of Dring and E-ring isoprostanes. J Biol Chem. 1994;269(6):4317-26.

27. Murphy RC, Johnson KM. Cholesterol, reactive oxygen species, and the formation of biologically active mediators. J Biol Chem. 2008;283(23):15521-5.

28. Jomova K, Valko M. Advances in metal-induced oxidative stress and human disease. Toxicology. 2011;283(2-3):65-87.

29. Gargalovic PS, Imura M, Zhang B. Identification of inflammatory gene modules based on variations of human endothelial cell responses to oxidized lipids. Proc Natl Acad Sci U S A. 2006;103(34):12741-6.

30. Hörkkö S, Bird DA, Miller E, et al. Monoclonal autoantibodies specific for oxidized phospholipids or oxidized phospholipidprotein adducts inhibit macrophage uptake of oxidized lowdensity lipoproteins. Clin Invest. 1999;103(1):117-28.

31. Lee H, Shi W, Tontonoz P, et al. Role for peroxisome proliferatoractivated receptor alpha in oxidized phospholipid-induced synthesis ofmonocyte chemotactic protein- 1 and interleukin- 8 by endothelial cells. Circ Res. 2000;87(6):516-21.

32. Loidl A, Sevcsik E, Riesenhuber G, et al. Oxidized phospholipids in minimally modified low density lipoprotein induce apoptotic signaling via activation of acid sphingomyelinase in arterial smooth muscle cells. J Biol Chem. 2003;278(35):32921-8.

33. Chatterjee S, Berliner JA, Subbanagounder GG, et al. Identification of a biologically active component in minimally oxidized low density lipoprotein (MM-LDL) responsible for aortic smooth muscle cell proliferation. Glycoconj J. 2004;20(5):331-8.

34. Bochkov VN, Mechtcheriakova D, Lucerna M, et al. Oxidized phospholipids stimulate tissue factor expression in human endothelial cells via activation of ERK/EGR-1 and $\mathrm{Ca}(++) / \mathrm{NFAT}$. Blood. 2002;99(1):199-206.

35. Subbanagounder G, Deng Y, Borromeo C, et al. Hydroxy alkenal phospholipids regulate inflammatory functions of endothelial cells. Vasc Pharmacol. 2002;38(4):201-9.

36. Moore KP, Darley-Usmar V, Morrow J, Roberts 2nd LJ. Formation of F2-isoprostanes during oxidation of human low-density lipoprotein and plasma by peroxynitrite. Circ Res. 1995;77(2):335-41

37. Lynch SM, Morrow JD, Roberts 2nd LJ, Frei B. Formation of noncyclooxygenase-derived prostanoids (F2-isoprostanes) in plasma and low density lipoprotein exposed to oxidative stress in vitro. $\mathrm{J}$ Clin Invest. 1994;93(3):998-1004.

38. Morrow JD, Awad JA, Boss HJ, et al. Non-cyclooxygenasederived prostanoids (F2-isoprostanes) are formed in situ on phospholipids. Proc Natl Acad Sci U S A. 1992;89(22):10721-5.

39. Praticò D, Iuliano L, Mauriello A, et al. Localization of distinct F2isoprostanes in human atherosclerotic lesions. J Clin Invest. 1997;100(8):2028-34.

40. Watson AD, Subbanagounder G, Welsbie DS, et al. Structural identification of a novel pro-inflammatory epoxyisoprostane phospholipid in mildly oxidized low density lipoprotein. J Biol Chem. 1999;274(35):24787-98.

41. Birukov KG, Bochkov VN, Birukova AA, et al. Epoxycyclopentenonecontaining oxidized phospholipids restore endothelial barrier function via Cdc42 and Rac. Circ Res. 2004;95(9):892-901.

42. Poliakov E, Meer SG, Roy SC, et al. Iso[7]LGD2-protein adducts are abundant in vivo and free radical-induced oxidation of an arachidonyl phospholipid generates this D series isolevuglandin in vitro. Chem Res Toxicol. 2004;17(5):613-22.

43. Roberts 2nd LJ, Fessel JP. The biochemistry of the isoprostane, neuroprostane, and isofuran pathways of lipid peroxidation. Chem Phys Lipids. 2004;128(1-2):173-86.

44. Levy R. The role of cytosolic phospholipase a2-alfa in regulation of phagocytic functions. Biochim Biophys Acta. 2006;1761(11):1323-34.

45. Triggiani M, Granata F, Giannattasio G, Marone G. Secretory phospholipases a2 in inflammatory and allergic diseases: not just enzymes. J Allergy Clin Immunol. 2005;116(5):1000-6.
46. Dioszeghy V, Rosas M, Maskrey BH, et al. 12/15-Lipoxygenase regulates the inflammatory response to bacterial products in vivo. $\mathrm{J}$ Immunol. 2008;181(9):6514-24.

47. Kondo M, Tamaoki J, Takeyama K, et al. Interleukin-13 induces goblet cell differentiation in primary cell culture from Guinea pig tracheal epithelium. Am J Respir Cell Mol Biol. 2002;27(5):536-41.

48. Kondo M, Tamaoki J, Takeyama K, et al. Elimination of IL-13 reverses established goblet cell metaplasia into ciliated epithelia in airway epithelial cell culture. Allergol Int. 2006;55(3):329-36.

49. Morcillo EJ, Cortijo J. Mucus and MUC in asthma. Curr Opin Pulm Med. 2006;12(1):1-6.

50. Brown CD, Kilty I, Yeadon M, Jenkinson S. Regulation of 15lipoxygenase isozymes and mucin secretion by cytokines in cultured normal human bronchial epithelial cells. Inflamm Res. 2001;50(6):321-6.

51. Zhao J, Maskrey B, Balzar S, et al. Interleukin-13-induced MUC5AC is regulated by 15-lipoxygenase 1 pathway in human bronchial epithelial cells. Am J Respir Crit Care Med. 2009;179(9):782-90.

52. Zhao J, O'Donnell VB, Balzar S, et al. 15-Lipoxygenase 1 interacts with phosphatidylethanolamine-binding protein to regulate MAPK signaling in humanairway epithelial cells. Proc Natl Acad Sci U S A. 2011;108(34):14246-51.

53. • Uderhardt S, Herrmann M, Oskolkova OV, et al. 12/15-lipoxygenase orchestrates the clearance of apoptotic cells and maintains immunologic tolerance. Immunity. 2012;36(5):834 46. This elegant study shows the involvement of 12/15-lipoxygenase in the regulation of apoptotic cell clearance and maintenance of self-tolerance.

54. Maskrey BH, Bermúdez-Fajardo A, Morgan AH, et al. Activated platelets and monocytes generate four hydroxyphosphatidylethanolamines via lipoxygenase. J Biol Chem. 2007;282(28):20151-63.

55. Miller YI, Chang MK, Funk CD, et al. 12/15-lipoxygenase translocation enhances site-specific actin polymerization in macrophages phagocytosing apoptotic cells. J Biol Chem. 2001;276(22):19431-9.

56. von Schlieffen E, Oskolkova OV, Schabbauer G, et al. Multi-hit inhibition of circulating and cell-associated components of the tolllike receptor 4 pathway by oxidized phospholipids. Arterioscler Thromb Vasc Biol. 2009;29(3):356-62.

57. Huang JT, Welch JS, Ricote M, et al. Interleukin-4-dependent production of ppar-gamma ligands in macrophages by $12 / 15$ lipoxygenase. Nature. 1999;400:378-82.

58. Yeung J, Holinstat M. 12-lipoxygenase: a potential target for novel anti-platelet therapeutics. Cardiovasc Hematol Agents Med Chem. 2011;9(3):154-64.

59. Dogné JM, de Leval X, Delarge J, et al. New trends in thromboxane and prostacyclin modulators. Curr Med Chem. 2000;7(6):609-28.

60. Weinstein EA, Li H, Lawson JA, et al. Prothrombinase acceleration by oxidatively damaged phospholipids. J Biol Chem. 2000;275(30):22925-30.

61. Pickering W, Gray E, Goodall AH, Barrowcliffe TW. Effects of apoptosis and lipid peroxidation on T-lymphoblastoid phospholipid-dependent procoagulant activity. J Thromb Haemost. 2008;6(7):1122-30.

62. Kozak KR, Marnett LJ. Oxidative metabolism of endocannabinoids. Prostaglandins Leukot Essent Fat Acids. 2002;66(2-3):211-20.

63. Kozak KR, Crews BC, Morrow JD, et al. Metabolism of the endocannabinoids, 2-arachidonylglycerol and anandamide, into prostaglandin, thromboxane, and prostacyclin glycerol esters and ethanolamides. J Biol Chem. 2002;277(47):44877-85.

64. Rouzer CA, Marnett LJ. Glycerylprostaglandin synthesis by resident peritoneal macrophages in response to a zymosan stimulus. $\mathrm{J}$ Biol Chem. 2005;280(29):26690-700.

65. Soehnlein O. Multiple roles for neutrophils in atherosclerosis. Circ Res. 2012;110(6):875-88.

66. Döring Y, Drechsler M, Wantha S, et al. Lack of neutrophilderived CRAMP reduces atherosclerosis in mice. Circ Res. 2012; 110(8):1052-6. 